\title{
What should define a SARS-CoV-2 "breakthrough" infection?
}

\author{
John S. Schieffelin, ${ }^{1}$ Elizabeth B. Norton, ${ }^{2}$ and Jay K. Kolls ${ }^{3}$ \\ ${ }^{\top} T u l a n e$ Department of Pediatrics, ${ }^{2}$ Tulane Department of Microbiology and Immunology, and ${ }^{3}$ Center for Translational Research in Infection and Inflammation, Tulane School of Medicine, New Orleans, \\ Louisiana, USA.
}

$\mathbf{R}$ reports of SARS-CoV-2 "breakthrough" infections that have occurred in recipients of the FDA-approved SARS-CoV-2 vaccines (1). These reports have the potential to undermine public confidence in the efficacy of these vaccines. Moreover, the use of the term "breakthrough" infections implies that the virus broke through a protective barrier provided by the vaccine. But is this what happened in these cases? In most cases, the answer is no, and this answer lies in the fundamental understanding of the mucosal immune system throughout the respiratory tract: the upper respiratory tract and the lower respiratory tract. The current vaccines prevent COVID-19, by definition a lower respiratory tract illness, in greater than $90 \%$ of recipients $(2-5)$. Recent studies of fully vaccinated individuals monitored by routine nasal testing have shown marked reductions, but not a complete absence, of SARS-CoV-2-positive tests (6-9). We believe it is a mistake to think that these vaccines will prevent nasal (upper airway) infection. This inference is based on the current routes of immunization.

\section{Immune response to vaccination}

The current vaccines elicit anti-spike IgG as well as $\mathrm{T}$ cell responses that can be detected in peripheral blood. However, there is a paucity of data on whether these intramuscular vaccines elicit respiratory tract-specific immune responses such as generation of tissue-resident memory B cells (10) or T cells (11). In fact, this is highly unlikely. Lessons from conjugated
Streptococcus pneumoniae vaccines show strong effects in reducing meningitis and, to a certain extent, pneumonia because of $S$. pneumoniae serotypes in the vaccine (12). However, these vaccines do not completely prevent nasal colonization by S. pneumoniae (13). Prior clinical experience with the anti-respiratory syncytial virus (anti-RSV) monoclonal antibody (an IgG1 isotype) had little effect on RSV infection of the upper respiratory tract but prevented RSV hospitalization for lower respiratory tract infection (14). Similarly, the seasonal inactivated influenza vaccine delivered intramuscularly protects individuals against acute respiratory illness and is associated with high levels of virus-neutralizing serum antibodies, but does not block viral transmission as observed in cohorts that included household contacts (15). On this basis, we would predict that systemic IgG would have little effect on nasal infection, nasal carriage, or, more importantly, nasal shedding of virus. Hence, vaccination with the currently approved vaccines will not eliminate the need to continue some mitigation procedures such as mask wearing and social distancing in highdensity-contact scenarios if high vaccination rates to achieve herd immunity are not reached (16).

\section{Expectations regarding effective vaccines}

We have not done a good job of communicating to the press or the public the concept that limited nasal infection may occur in vaccinated individuals. Understanding the regional aspects (upper
Conflict of interest: JKK and EBN are named inventors on the patent application PCT/US20/25518 ("Multivalent vaccines derived from klebsiella out membrane proteins"). JKK is a named inventor on a biologic that may have utility in treating or preventing COVID-19 in high-risk groups (PCT/US21/29121; "Compositions and methods for preventing or reducing the effects of infections by coronaviruses that bind the extracellular domain of the ACE2 receptor). Copyright: () 2021, American Society for Clinical Investigation.

Reference information: J Clin Invest. 2021;131(12):e151186. https://doi.org/10.1172/JCI151186. versus lower respiratory tract) of vaccine effectiveness are key to ensure public confidence in the vaccines as well as to help the public understand the need to continue adhering to public health mitigation recommendations. In other words, we should expect SARS-CoV-2 vaccines to prevent disease, but without a transmission-blocking vaccine or achieving herd immunity, we cannot expect to vaccinate our way back to $100 \%$ of our pre-pandemic activities. Moreover, this highlights the need to also fund basic research to develop safe mucosal vaccines that can generate tissueresident memory $\mathrm{B}$ and $\mathrm{T}$ cells in the respiratory mucosa that may be more effective in mediating sterilizing immunity in the respiratory tract. In fact, a recent study using mucosal adenovirus vaccines appeared superior to intramuscular routes in controlling viral load in the respiratory tract (17).

\section{Defining breakthrough infections}

We need a more precise definition of what a true breakthrough case is (Figure 1). We suggest that breakthrough disease should be defined by a positive SARS-CoV-2 PCR test from the respiratory tract and documentation of lower respiratory tract disease (Figure 1). On the other hand, detection of the SARS-CoV-2 virus in the upper respiratory tract in a fully vaccinated individual with no symptoms or mild upper respiratory tract infection symptoms (loss of smell) would not meet the criteria for a breakthrough infection. This is not to say that these positive tests are not infections, as they do carry the risk of transmission, they are just not "breakthrough" infections. Perhaps they are best classified as post-vaccination upper respiratory tract infections. The formal criteria for a true breakthrough infection could include evidence of radiographic abnormalities, a 


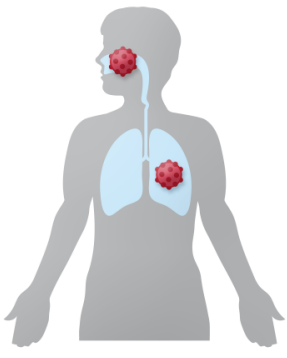

Vaccine nonresponder $\left(\mathrm{PCR}^{+}, \mathrm{LRI}\right)$

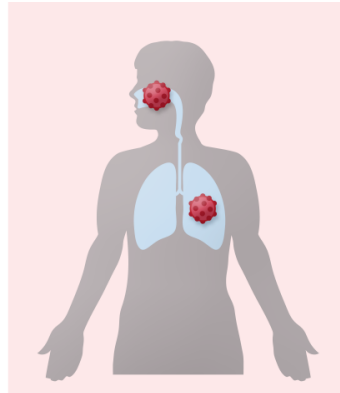

Vaccine poor-responder/ ineffective responses to new variant $\left(\mathrm{PCR}^{+}, \mathrm{LRI}\right)$

Variable

Variable

Positive

Yes

High

Clinical disease

Yes

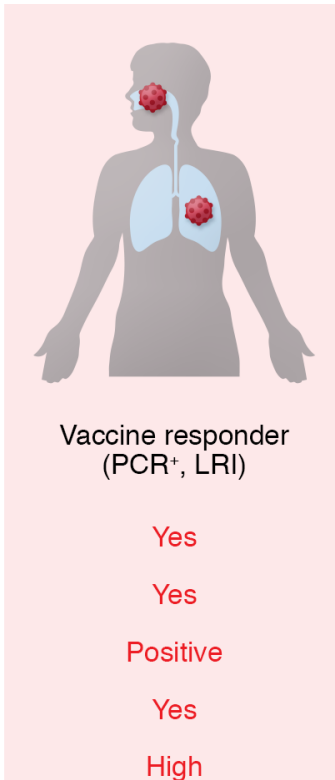

Clinical disease

Yes

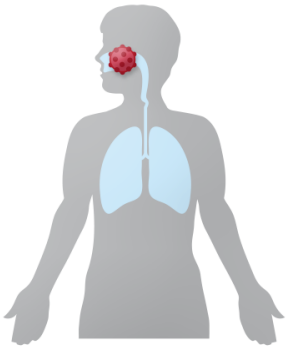

Vaccine responder (PCR+ ${ }^{+}$asymptomatic)

$$
\begin{gathered}
\text { Yes } \\
\text { Yes } \\
\text { Positive } \\
\text { No }
\end{gathered}
$$

Low to moderate*

Asymptomatic/URI only

No

Figure 1. Clinical scenarios and proposed classification of breakthrough infection based on disease in the lower respiratory tract. We propose that breakthrough infection after COVID-19 vaccination is defined by evidence of lower respiratory tract infection (LRI) and a positive PCR test for SARS-COV-2. Vaccinated individuals who are asymptomatic or have symptoms limited to an upper respiratory tract infection (URI) are not experiencing a breakthrough infection, although it is important to understand that these individuals may still be able to transmit virus. We also suggest that those individuals who do not mount an effective immune response to vaccination do not represent cases of breakthrough infection. * Transmission risk is likely proportionate to the viral copy number.

widened alveolar-arterial (A-a) gradient, or hypoxemia defined by low oxygen saturations in the arterial blood by pulse oximetry. These criteria will be critical to determine which positive SARS-CoV-2 tests are true vaccine failures in the lower respiratory tract versus the upper respiratory tract. In the current environment of widespread vaccine hesitancy, these distinctions will be crucial to maintaining, and possibly increasing, the public's trust in vaccines as well as understanding the role of vaccination in ending the pandemic.

\section{Acknowledgments}

The authors acknowledge research support from the NIH (U54-CA260581) for this work.

Address correspondence to: Jay K. Kolls, Professor of Medicine and Pediatrics, John W. Deming Endowed Chair in Internal Medicine, Center for Translational Research in Infection and Inflammation, Tulane School of Medicine, JBJ 375, 333 S. Liberty St, New Orleans, Louisiana 70112, USA. Phone: 504.988.0456; Email: jkolls1@tulane.edu.
1. Hacisuleyman E, et al. Vaccine breakthrough infections with SARS-CoV2 variants [published online April 21, 2021]. N Engl J Med. https://doi. org/10.1056/NEJMoa2105000.

2. Polack FP, et al. Safety and efficacy of the BNT162b2 mRNA Covid-19 vaccine. $N$ EnglJ Med.2020;383(27):2603-2615.

3. Baden LR, et al. Efficacy and safety of the mRNA-1273 SARS-CoV2 vaccine. $N$ Engl J Med. 2021;384(5):403-416.

4. Sadoff J, et al. Interim results of a phase 1-2a trial of Ad26.COV2.S Covid-19 vaccine [published online January 13, 2021]. N Engl J Med. https:// doi.org/10.1056/NEJMoa2034201.

5 . Voysey M, et al. Single-dose administration and the influence of the timing of the booster dose on immunogenicity and efficacy of ChAdOx1 nCoV-19 (AZD1222) vaccine: a pooled analysis of four randomised trials. Lancet. 2021;397(10277):881-891.

6. Teran RA, et al. Postvaccination SARS-CoV2 infections among skilled nursing facility residents and staff members - Chicago, Illinois, December 2020-March 2021. MMWR Morb Mortal Wkly Rep. 2021;70(17):632-638.

7. Daniel W, et al. Early evidence of the effect of SARS-CoV2 vaccine at One Medical Center [published online March 23, 2021]. N Engl JMed. https://doi.org/10.1056/NEJMc2102153.

8. Keehner J, et al. SARS-CoV2 infection after vaccination in health care workers in California. N Engl JMed. 2021;384(18):1774-1775.

9. Thompson MG, et al. Interim estimates of vac- cine effectiveness of BNT162b2 and mRNA-1273 COVID-19 vaccines in preventing SARS-CoV2 infection among health care personnel, first responders, and other essential and frontline workers - eight U.S. locations, December 2020March 2021. MMWR Morb Mortal Wkly Rep. 2021;70(13):495-500.

10. Allie SR, et al. The establishment of resident memory B cells in the lung requires local antigen encounter. Nat Immunol. 2019;20(1):97-108.

11. Farber DL. Form and function for T cells in health and disease. Nat Rev Immunol. 2020;20(2):83-84.

12. Berical AC, et al. Pneumococcal vaccination strategies. An update and perspective. Ann Am Thorac Soc. 2016;13(6):933-944.

13. Green C, et al. A simple approach to pneumococcal vaccination in adults. J Glob Infect Dis. 2018;10(3):159-162.

14. No authors listed. Palivizumab, a humanized respiratory syncytial virus monoclonal antibody, reduces hospitalization from respiratory syncytial virus infection in high-risk infants. The IMpact-RSV Study Group. Pediatrics. 1998;102(3 Pt 1):531-537.

15. Ohmit SE, et al. Influenza vaccine effectiveness in the community and the household. Clin Infect Dis. 2013;56(10):1363-1369.

16. Aschwanden C. Five reasons why COVID herd immunity is probably impossible. Nature. 2021;591(7851):520-522.

17. Hassan AO, et al. A single-dose intranasal ChAd vaccine protects upper and lower respiratory tracts against SARS-CoV2. Cell. 2020;183(1):169-184. 